\title{
EVALUATING TESTER EXPERIENCE IN AN INDUSTRIAL ENVIRONMENT: AN EXPERIENCE REPORT
}

\author{
Camila Bernardon ${ }^{1}$, Oswald M. Ekwoge ${ }^{1}$, Thais E. Colares ${ }^{1}$, Bruno A. Bonifacio ${ }^{1}$, \\ Thiago L. Souto ${ }^{1}$, Marcelo A. Reis ${ }^{1}$, Andre S. Neto ${ }^{1}$ and Awdren de Lima Fontão ${ }^{2}$

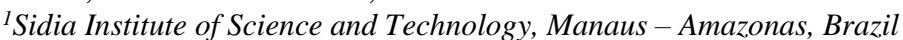 \\ ${ }^{2}$ Federal University of Mato Grosso do Sul-UFMS, Campo Grande - Mato Grosso do Sul, Brazil
}

\begin{abstract}
Software testing is essential to guarantee product quality. As such, while technologies, automation, and systematic methodologies are important, the human aspects of testing, that is, the individual performing the activity, certainly does matter in software testing. As a result, Tester Experience (TX) has been defined to bridge the gap between technical and human aspects of software testing. TX can identify influence factors that can affect test effectiveness from testers' perspective. In this paper, we present our experience, through previous results, where we evaluate TX in the Google Approval (GA) homologation team, with 27 subjects of SIDIA Institute of Science and Technology. The results show that TX successfully captured participants' experience with respect to their daily activities and artifacts. In addition to this, TX was also able to point out points for improvement within the GA team.
\end{abstract}

\section{KEYWORDS}

Tester Experience, TX, Experience Report

\section{INTRODUCTION}

The successful accomplishment of software development is surely a collaborative process. For this reason, it relies on the efforts of all practitioners involved in the process development, including managers, developers, and testers (Kanakis et al., 2019). In this case, software testing is an essential activity in software development to guarantee the time to market for new products, with quality (Romano et al., 2019). Thus, best practices and initiatives related to testing automation have been proposed to reduce cost and the participation of the human element in software activities. However, testing is a substantially human-based activity. Therefore, efficient ways of testing software products for quality assurance will require a better comprehensive understanding of testers' feelings, perceptions, and motivations, referred as Tester Experience - TX (Ekwoge et al., 2017).

TX can be defined as a means of capturing how testers think and feel about their activities within the software testing environment. TX uses the assumption that an improvement of the tester's experience has a positive impact on quality assurance (Ekwoge et al., 2017). For this reason, we choose this method to verify tester's perception of our homologation team, called Google Approval (GA). Thus, in this paper we report our experience to verify the TX of our GA team. This team is responsible to validate requirements from Google related security patches, user experience recommended to Open Handset Alliance partners.

We carried out in Sidia R\&D Institute, in the software homologation team, of the Mobile Product Software division. During the study, we collected opinions of 27 software testers themselves, using an online survey, on the importance TX during test activities. The survey responses strongly suggest that while testing tools, testing environment, and training are important, human factors are crucial for software testing to be effective.

The remainder of this paper is organized as follows: Sections 2 presents backgrounds and related concepts. Section 3 describes the planning, execution and results of the study. Finally, the considerations and suggestions for future work are presented in Section 4. 


\section{BACKGROUND}

Ekwoge et al., (2017) define TX as an approach derived from the other two methods: User Experience (UX) and Developer Experience (DX). While UX is a term that captures how people feel about products, systems and services (ISO 9241-210:2010, 2010) and DX is focused on how developers think and feel about their activities (Fagerholm and Munch, 2012), TX adapts the cited concepts to software testing.

TX concept is intended to abstract human characteristics and factors that are intuitive for testing practitioners to help them better understand, analyze, design and improve project environments with respect to testers' perceptions and feelings (Ekwoge et al., 2017). TX has three main factor categories that affect testers' experience: Cognition, Conation, Affection. Each of these three factors was divided into sub-factors (Figure 1).

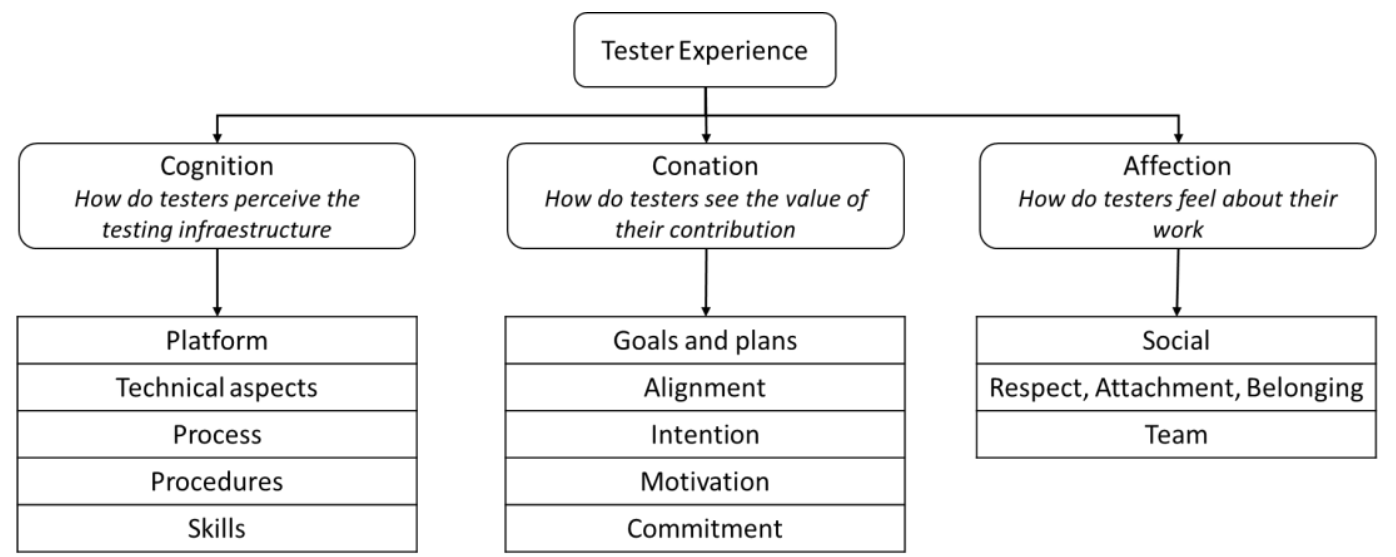

Figure 1. Factors and sub-factors of TX [Ekwoge et al., 2017]

Figure 1 shows factors relation and their sub-factors. These factors and sub-factors allow better understanding about TX influence, as follows:

a) Cognition: related to attention, memory, producing and understanding language, problem solving and decision making. This category consists of factors that affect how testers perceive their testing infrastructure on an intellectual level. Sub-factors: platform, technical aspects, process, procedures and skills.

b) Conation: related to impulse, desire, volition, striving. This category consists of factors that affect how testers see the value of their contribution. Sub-factors: goals and plans, alignment, intention, motivation, commitment.

c) Affection: related to feeling and emotions. This category consists of factors that influence how testers feel about their work as respect, social aspects. Sub-factors: social, respect, attachment, belonging, team.

Another important study is presented by Kanij et al. (2014). They conducted a preliminary study, through a survey, that collects the importance that human factors in test activities, focusing on the development of systematic, standardized, and automated testing methodologies and tools. The results suggest that although testing tools and training were considered, human factors need to be considered highly important as relates to testing activities.

Mostly the results of the studies were based on literature review and some qualitative methods like interviews, surveys, and diary studies [Fagerholm and Münch (2013); Fontão, Dias-Neto, and Viana (2017); Murphy et al. (2018); Dong and Khandwala (2019)]. As such, in order to better capture and evaluate testers' perceptions about their testing activities, we opted to apply TX within a specific team, more specifically, the Google Approval homologation team of Sidia R\&D Institute. Next Section we present the study design, execution and results.

\section{USING TX TO COLLECT TESTERS PERCEPTION: CASE STUDY}

Sidia is a R\&D Institute, located in Manaus-Amazonas, responsible for developing and testing innovative digital solutions for the global market. Its main partner is Samsung and the embedded software on these mobile devices are produced for the Latin American market (Barbosa et al. 2019). 
Software development process is based on release, where is added new requirements and security patches from Google platform. During release process, after the software is released, it must go through a homologation process with respect to Google's requirements which include security patches, User Interface (UI) requirements, and apps required by Google (Android Security Bulletin, 2020). During the homologation phase, a specific team is responsible for testing Google's requirements, applied in a software release version. This team, called the Google Approval (GA) team. It executes tests to guarantee that all Google requirements are applied. We choose to evaluate TX of GA during test execution of the homologation release process.

Goal: The goal of this study was to evaluate TX of the GA team with the aim to characterize with respect to factors affecting TX from testers' perspective in the context of a release homologation process.

Metrics: As main metric we use factors, and subfactors described detailed in Ekwoge et al., (2017). These factors were used to group questions to better collect TX perceptions from subjects.

Subjects: We chose 27 volunteer participants from Sidia's GA team. The subjects who agreed to participate in this study were assigned a consent form to keep their personal information secure. Among the subjects, $15 \%$ have been working with the GA team for less than one year, $30 \%$ for one year, $18 \%$ for two years and $37 \%$ have been working in the GA team for three years or more.

Procedure: We wanted to evaluate the TX of our GA homologation team, during test activities. To achieve this, we created an anonymous online questionnaire, with questions grouped by TX factors and sub-factors. We identify how each factor/sub-factor affects participants' TX by examining their activities within the testing process. Some of these activities include: resources (workstation, devices, cables, sim cards), documentation (Wiki, Confluence, Jira), tools. In addition to these activities, we collected information about the experience of these members on software testing and required software testing skills.

The study was carried out in 2020. The survey included different types of questions, e.g., multiple-choice, Likert-scale, and short answer questions. The survey had two main sections, grouping questions by topic to minimize the cognitive load on participants. We present the partial results of this survey in the next section.

\subsection{Results}

As earlier mentioned, TX is divided into three main factors categories: Cognition, Conation, and Affection. The cognition factor is related to technical perceptions about working tasks. Its sub-factors include: platform; technical aspects; skills; process, and procedures. The conation factor is a perspective that can offer value or morale to the software tester, during the testing process. Sub-factors in this category are alignment; motivation; goals and plans; commitment. Lastly, the affection factor is related to social interaction. Sub-factors are respect, attachment, and belonging; social aspects, and team.

As relates to the cognition factor, Figure 2 shows results obtained from the survey. As concerns platform, $49 \%$ of subjects strongly agreed that their workplace is comfortable during working days, $40 \%$ agreed and $11 \%$ disagreed or were neutral. With respect to resources available to get their job done, $77 \%$ strongly agreed or agreed that resources are available and adequate during the homologation process. Finally, 96\% considered the infrastructure provided as satisfying. Only $4 \%$ reported that there is a need for improvement related to platform. The participants related the lack of documentation; still, others related that internet connection needs to improve to optimize test duration.

As relates to procedures and testing specifications, $37 \%$ of subjects reported that they experience some difficulties executing procedures in the GA team. $77 \%$ consider their execution of procedures acceptable during testing execution. Finally, 92\% considered the execution of procedures relevant for the release process. With respect to the process sub-factor, $77 \%$ felt comfortable executing tests following the GA process, $18 \%$ felt neutral about, or disagreed with, the GA process. In addition, $71 \%$ related having sufficient information about GA process. On other hand, 59\% reported that need more information about GA process. This result can be explained by the arrival of new members to the team, who are still learning the GA process.

Considering technical aspects, $60 \%$ related that tools generate safe results during testing execution, and $34 \%$ were neutral; another $6 \%$ disagreed. About documentation, $60 \%$ answered that documentation is useful to learn about the GA process. About automated tools, $60 \%$ related that tools are important to improve their tests execution. Finally, $89 \%$ felt comfortable using tools available to perform their testing activities. About skills, 60\% reported that they need more information and knowledge about GA activities. So as improvement, GA needs to offer more trainings related to GA tests. And 97\% reported that they have the skills required to execute GA tests. At the end of this part, we asked the participants whether a better experience in all aspects and activities related to cognation can improve their TX. $81 \%$ agreed, $4 \%$ disagreed and $15 \%$ were not sure. 
Figure 3 shows sub-factors included in the Conation factor. As relates to goals and plans, $99 \%$ of subjects reported that the GA team is important in the release process. As concerns the importance of their contribution, $67 \%$ reported that they consider their activities an important contribution to the GA process. In addition, $72 \%$ the subjects that the GA team contributes as success of projects and $83 \%$ consider their work as good as other R\&D partners. With respect to goal alignment with the GA team, $73 \%$ reported that their objectives are aligned with GA team members.

About commitment, $100 \%$ of subjects reported that are flexible to work in collaboration with other team members, 39\% reported that respect and problem solving are key to software testing. And 98\% reported that they are open to share knowledge with other colleagues. Concerning intention, $97 \%$ maintain a positive attitude during test process. Finally, as relates to motivation, $78 \%$ feel dedicated and committed to the team.

At the end of this section, we asked participants whether the value of their contribution (conation) can improve their TX. $96 \%$ agreed, while $4 \%$ were not sure.

Considering the Affection factor, Figure 4 shows the result for each related sub-factor. As concerns the team sub-factor, $78 \%$ feel that there is trust within the GA team. And $78 \%$ related that the there is a collaborative environment within the GA team. In addition, $67 \%$ feel that the environment is favorable and open to opportunities.

As relates to the respect, attachment and belonging sub-factor $86 \%$ feel mutual respect in the GA team. With respect to the social aspects sub-factor, $89 \%$ reported that they feel motivated and dedicated to the team's goal. $68 \%$ are open to new experiences. Finally, $70 \%$ feel committed and motivated to carry out activities in the GA team. We used these results to improve some issues found with this study.

At the end of this section, participants were asked whether the elements related to affection can improve their TX. 100\% agreed. In the next section, we present a final discussion and considerations.

\section{DISCUSSION AND FINAL CONSIDERATIONS}

The results obtained from this study clearly prove that TX is capable of capturing testers' perceptions with respect to the testing infrastructure, the value of their contribution, and how they feel about their work. With respect to cognition, the activity that received the most negative feedback from participants was the availability of adequate resources to perform their tasks. For instance, one participant said, "Sim card resources must be available to all team members. At the moment, some team members will have to be lucky to get a sim card which attends to all testing necessities. Some people have to suspend their activities until a sim card is available. Ideally, all network provider sim cards should be able to execute all telephone faults." Another participant said, "a better documentation with more details can improve the use of testing infrastructure." Still, other participants said, "I think some documentation could be more detailed." "Automation on common tasks like reviews and execution of Linux tests. Maybe more resources like good sim cards." It is necessary to invest in a favorable environment to carry out the tests, more equipment to improve to improve the GPS signals. Some cell phone models are not enough to deliver the result in a decent time. More cables, more sim cards and improvement in the quality of Linux machines to support the number of phones we use to run Linux tests. "Increase in the general internet quality, more automations, more maintenance of already available tools, more technical documentation." "I suggest for the development team to have a documentation to explain all details for the testers to use all the potentials of the automation tools. Sometimes, there are some functions that are not known by the team and it's something that could help in the process." "I believe that the most import aspect is discipline in the mode of operation. All the steps and activities to be executed should be well defined, and how to act when met with adverse situations. Documentation is one of the fundamental part of this process. However, good and profound training will facilitate the process as a whole and reduce the risk of rework."

With respect to the conative factor, the sub-factors that stood out were commitment and motivation. For instance, one participant said, "If we are more motivated and believe we are part of something bigger, we are all willing to do more and do better to contribute to the end goal." Still another said "Motivation and commitment are essential for good performance in our activities." Others mentioned: "The more we are motivated, the happier we are in performing our activities. We become committed to the team, achieve things together and therefore, obtain the best results in all we do." "People that are motivated in their job can perform better tests." Another tester believes communication is the key to obtaining better results: "I believe that communication is a skill that is too much important to engage all team members into a specific plan. It is important to settle ideas and organize tasks." 
In terms of value of their contribution, some testers said: "I believe that working with the GA team has attributed to me great professional value. Therefore, I have become dedicated to the team and more organized. I respect colleagues and superiors, and always search for more knowledge about GA's procedures in documentations. This is so important for the work I develop. Maintain a collective spirit and attend to all work demands with quality is essential for GA. All these mentioned values have granted me this experience." "I thank the GA team for making me a better tester because the collaboration with each other is amazing."

Another tester highlighted alignment as important in team engagement: "It is fundamental to always have alignment among team members, whether in terms of operation or motivation. A team with motivated members always tends to avoid rework, and are committed to completing their tasks in the best possible way." Another tester mentioned that team engagement can lead to the delivery of quality results: "Maintaining the team engaged during a project execution offers a sense of security and certitude that the project is delivered in time and in good quality."

Some testers believe continuous learning should be a point of improvement for the GA team: "Courses should be given for professional growth." "I am not stuck in a GA process. If I only execute the tests sent to me, I will never improve my experience as a tester. So I always seek for new knowledge, improving my skills. I also think good knowledge in Android, how it works, components, activities, intents, layouts, architecture, and log analysis will contribute to solving issues more quickly and easily. This gives us a better vision of the global process right to the final release phase...". Even though participants believe that the necessary infrastructure and resources are important to perform their testing activities, not everyone thinks it cognition is important for improving TX. In other words, testers believe cognitive factors are important in their testing activities, but not necessarily in improving TX.

\section{ACKNOWLEDGEMENT}

Our thanks to Sidia team and SAMSUNG Eletronics of Amazon Ltda, in the terms of Law N ${ }^{\circ} 8387 / 91$.

\section{REFERENCES}

Dong, T., \& Khandwala, K. (2019). The impact of cosmetic changes on the usability of error messages. In Extended abstracts of the 2019 chi conference on human factors in computing systems (LBW0273). ACM.

Ekwoge, O. M., Fontão, A., \& Dias-Neto, A. C. (2017). Tester experience: Concept, issues and definition. In 2017 ieee 41 st annual computer software and applications conference (compsac) (Vol. 1, pp. 208-213). IEEE

Fagerholm F, Munch J. Developer Experience : Concept and Definition. Int. Conf. Softw. Syst. Process (ICSSP '12), 2012, p. 73-7.

Fontão, A., Dias-Neto, A., \& Viana, D. (2017). Investigating factors that influence developers' experience in mobile software ecosystems. In 2017 ieee/acm joint 5th international workshop on software engineering for systems-ofsystems and 11th workshop on distributed software development, software ecosystems and systems-of-systems (jsos) (pp. 55-58). IEEE.

Google, "Android Security Features | Android Open Source Project," 2019. [Online]. Available: https://source.android.com/security/features. [Accessed: 13-Feb-2020].

H. O. B. Hualter O. Barbosa, B. A. Bonifacio, T. M. Menezes, L. F. Uebel, F. B. Pires, and A. F. Neto, "Uma Análise Do Uso De Ferramentas Em Desenvolvimento Distribuído De Software Para Atualização Da Plataforma Android," pp. 11-18, 2019, doi: 10.33965/ciawi2019_2019141002.

ISO9241-210:2010. Ergonomics of human system interaction - Part 210: Human-centered design for interactive systems. Switzerland 2010

Kanij T, Merkel R, Grundy J. A Preliminary Survey of Factors Affecting Software Testers. Aust. Softw. Eng. Conf., 2014c, p. 116-25.

Murphy, L., Kery, M. B., Alliyu, O., Macvean, A., \& Myers, B. A. (2018). Api designers in the field: Design practices and challenges for creating usable apis. In 2018 ieee symposium on visual languages and human-centric computing (vl/hcc) (pp. 249-258). IEEE. 